\title{
Pilot Matrix Design for Estimating Cascaded Channels in Two-Hop MIMO Amplify-and-Forward Relay Systems
}

\author{
Ma, J.; Orlik, P.; Zhang, J. Li, G.Y.
}

TR2011-061 June 2011

\begin{abstract}
In this paper, we consider a two-hop multi-inputmulti-output (MIMO) amplify- and-forward (AF) relay system consisting of a source node (SN), a destination node (DN), and a relay node $(\mathrm{RN})$ that simply amplifies and forwards its received signal to the DN without any further processing. In this system, the overall channel from the SN to the DN is a cascade of the backward relay channel over the SN-RN hop, the amplifying matrix at the RN, and the forward relay channel over the RNDN hop. We investigate the estimation of the two cascaded relay channels at the $\mathrm{DN}$ based on the predefined amplifying matrix applied at the $\mathrm{RN}$ and the corresponding overall channel obtained through the conventional channel estimation algorithms with the help of pilots transmitted by the SN. In particular, we find necessary and sufficient conditions on the pilot amplifying matrix sequence at the RN to ensure feasible relay channel estimation at the DN. Based on these conditions, we present rules to design diagonal or quasi-diagonal pilot amplifying matrices so that the cascaded relay channels can be estimated with minimum complexity at the RN. In the presence of imperfect overall channel state information at the DN, we further develop the approximate linear least-square estimation of the relay channels based on the designed pilot matrix sequence and demonstrate its performance by simulation results.
\end{abstract}

IEEE Transactions on Wireless Communications

This work may not be copied or reproduced in whole or in part for any commercial purpose. Permission to copy in whole or in part without payment of fee is granted for nonprofit educational and research purposes provided that all such whole or partial copies include the following: a notice that such copying is by permission of Mitsubishi Electric Research Laboratories, Inc.; an acknowledgment of the authors and individual contributions to the work; and all applicable portions of the copyright notice. Copying, reproduction, or republishing for any other purpose shall require a license with payment of fee to Mitsubishi Electric Research Laboratories, Inc. All rights reserved. 



\title{
Pilot Matrix Design for Estimating Cascaded Channels in Two-Hop MIMO Amplify-and-Forward Relay Systems
}

\author{
Jun Ma, Philip Orlik, Jinyun Zhang, and Geoffrey Ye Li
}

\begin{abstract}
In this paper, we consider a two-hop multi-inputmulti-output (MIMO) amplify-and-forward (AF) relay system consisting of a source node (SN), a destination node (DN), and a relay node (RN) that simply amplifies and forwards its received signal to the DN without any further processing. In this system, the overall channel from the $S N$ to the $D N$ is a cascade of the backward relay channel over the $\mathrm{SN}-\mathrm{RN}$ hop, the amplifying matrix at the RN, and the forward relay channel over the RNDN hop. We investigate the estimation of the two cascaded relay channels at the DN based on the predefined amplifying matrix applied at the $\mathrm{RN}$ and the corresponding overall channel obtained through the conventional channel estimation algorithms with the help of pilots transmitted by the $\mathrm{SN}$. In particular, we find necessary and sufficient conditions on the pilot amplifying matrix sequence at the $\mathrm{RN}$ to ensure feasible relay channel estimation at the DN. Based on these conditions, we present rules to design diagonal or quasi-diagonal pilot amplifying matrices so that the cascaded relay channels can be estimated with minimum complexity at the $\mathrm{RN}$. In the presence of imperfect overall channel state information at the $D N$, we further develop the approximate linear least-square estimation of the relay channels based on the designed pilot matrix sequence and demonstrate its performance by simulation results.
\end{abstract}

Index Terms-Amplify-and-forward, multi-input-multi-output, relay channel estimation, two-hop transmission

\section{INTRODUCTION}

Wireless relay has become a hot research topic in wireless communications. As a repeater, a wireless relay node $(\mathrm{RN})$ forwards signals from the source node ( $\mathrm{SN}$ ) to the destination node (DN). Wireless relay is suitable for realizing long-range communication or boosting the signal strength in coverage holes, such as in thick buildings or underground tunnels, or on the cell edge, etc [1]-[3]. Such wireless RN's also provide flexibility in meeting temporary communication demands under certain scenarios. Furthermore, the coordination between the SN and one or more RN's can be utilized to achieve spatial diversity to enhance the reliability or the capacity of wireless links [4]-[7].

Depending on how much signal processing is performed at the RN, the existing relay techniques can be broadly categorized as decode-and-forward (DF) and amplify-and-forward (AF) [8]. In the DF scheme, the RN detects and demodulates its received signals, decodes the encoded data, re-modulates the data, and forwards them to the DN. In contrast, the RN operating in AF mode only amplifies and forwards its received

This paper was presented in part at the IEEE International Conference on Communications (ICC), Dresden, Germany, June 2009. signals without any further processing and hence has much simpler implementation than that in DF mode. Furthermore, the $\mathrm{RN}$ in $\mathrm{AF}$ mode does not need any a priori information of its received signal and thus can be applied to any scenario.

In this paper, we consider a narrowband two-hop multiinput-multi-output (MIMO) AF relay system in which there is no direct communication link between the SN and the DN and data are conveyed from the $\mathrm{SN}$ to the DN via two cascaded orthogonal channels by frequency-division or time-division. In this system, the overall channel between the SN and the DN is a cascade of the backward relay channel over the SN-RN hop, the amplifying matrix at the RN, and the forward relay channel over the RN-DN hop. With the help of pilots inserted at the $\mathrm{SN}$, the overall channel from the $\mathrm{SN}$ to the DN can be obtained at the DN through the conventional channel estimation algorithms that have been proposed for conventional MIMO systems [9]-[12]. Recently, special overall channel estimation algorithms have been also proposed for AF-based cooperative communication systems in [13] and [14]. While the overall channel state information (CSI) guarantees feasible data detection at the DN, the involved relay CSI, if available, can be utilized to improve the overall system performance. Since the noise at the $\mathrm{RN}$ is amplified and forwarded to the DN together with the signal, the forward relay CSI over the RN-DN hop can be utilized to estimate the correlation matrix of the overall noise vector at the DN for system performance improvement. Conventionally, the relay channels over the SN-RN and RNDN hops can be estimated directly at the RN and the DN with the help of pilots inserted at the SN and the RN, respectively. However, such direct relay channel estimation is based on the assumption that the $\mathrm{RN}$ is aware of the structure of its received signal and capable of performing further signal processing. In practice, it is attractive to keep minimum complexity at the RN that is supposed to be cost-efficient and undertakes no task except simply amplifying and forwarding its received signals. In this paper, we extend our prior work in [15] and investigate estimating the two cascaded relay channels at the DN based on a predefined pilot amplifying matrix sequence at the $\mathrm{RN}$ and the corresponding overall channel sequence obtained at the DN through conventional channel estimation algorithms. In particular, we find necessary and sufficient conditions on the pilot amplifying matrix sequence at the RN to ensure feasible relay channel estimation at the DN. Based on these conditions, we derive rules to design diagonal or quasi-diagonal pilot amplifying matrices so that the relay channels can be estimated with minimum complexity 


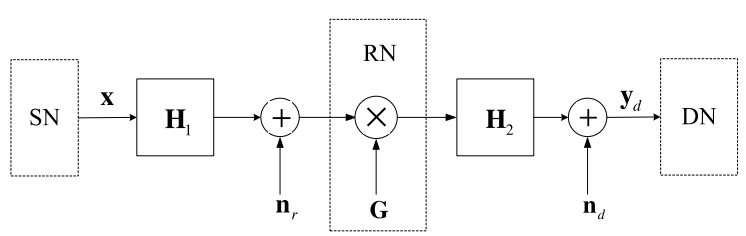

Fig. 1. Two-hop MIMO AF relay system model

at the RN. In the presence of imperfect overall CSI at the $\mathrm{DN}$, we further develop the approximate linear least-square (LS) estimation of the relay channels based on the designed pilot matrix sequence and demonstrate its performance by simulation results. Furthermore, initial simulation results are also presented to demonstrate the performance improvement of the two-hop MIMO AF relay system achieved by relay channel estimation at the DN.

The remainder of this paper is organized as follows. In Section II, we present the system model and the principle of estimating the cascaded relay channels at the DN. In Section III, we investigate necessary and sufficient conditions for feasible relay channel estimation. In Section IV, we derive rules to design diagonal or quasi-diagonal pilot amplifying matrices meeting these conditions. In Section V, we develop approximate linear LS estimation of the relay channels in the presence of imperfect overall CSI. In Section VI, we extend relay channel estimation to a general two-hop MIMO AF system. In Section VII, we present simulation results on the approximate linear LS relay channel estimation in a noisy environment. Finally we conclude this paper in Section VIII.

\section{Problem Formulation}

In this paper, we are concerned with a narrowband twohop MIMO AF relay system, which can be directly used in a broadband multicarrier system like orthogonal frequency division multiplexing (OFDM). The block diagram of such a system is shown in Fig. 1. As indicated, this system consists of an SN, an RN, and a DN, all of which utilize multiple transmit/receive antennas. In this system, there is no direct communication link between the SN and the DN and data are conveyed from source to destination via two cascaded orthogonal channels by frequency-division or time-division.

\section{A. System Model}

Suppose that the number of antennas at the SN, the RN, and the DN are $N_{s}, N_{r}$, and $N_{d}$, respectively. Denote $\mathbf{H}_{1}$ as the $N_{r} \times N_{s}$ backward relay channel matrix between the $\mathrm{SN}$ and the RN, $\mathbf{H}_{2}$ as the $N_{d} \times N_{r}$ forward relay channel matrix between the RN and the DN, both of which are assumed nonsingular throughout this paper, and $\mathbf{G}$ as the $N_{r} \times N_{r}$ amplifying matrix at the $\mathrm{RN}$, and then the received signal vector at the $\mathrm{DN}$ is given by

$$
\mathbf{y}_{d}=\mathbf{H}_{2} \mathbf{G H}_{1} \mathbf{x}+\mathbf{H}_{2} \mathbf{G} \mathbf{n}_{r}+\mathbf{n}_{d}=\mathbf{H x}+\mathbf{n},
$$

where $\mathbf{x}$ denotes the transmitted signal vector of the $\mathrm{SN}$, and $\mathbf{n}_{r}$ and $\mathbf{n}_{d}$ denote the local noise vectors at the RN and the DN, respectively. In (1), $\mathbf{H}$ denotes the $N_{d} \times N_{s}$ overall channel

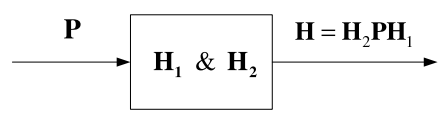

Fig. 2. Equivalent system for estimating cascaded relay channels at the DN

matrix between the SN and the DN defined as $\mathbf{H}=\mathbf{H}_{2} \mathbf{G H}_{1}$, and $\mathbf{n}$ denotes the overall noise vector at the DN defined as $\mathbf{n}=$ $\mathbf{H}_{2} \mathbf{G n}_{r}+\mathbf{n}_{d}$, which consists of the colored noise forwarded from the RN and the local white noise at the DN. In order for the overall channel to be nonsingular, we assume $N_{r} \geq$ $\min \left\{N_{s}, N_{d}\right\}$ throughout this paper. Furthermore, to maintain a constant power amplifying gain at the RN, we let $\mathbf{G}=g \mathbf{P}$ where $g$ is the fixed amplifying gain of the RN and $\mathbf{P}$ is a unitary matrix. In practice, $\mathbf{P}$ is usually a diagonal matrix or a permutation of a diagonal matrix, also called a quasi-diagonal matrix in this paper, so as to reduce the complexity in the matrix multiplication manipulation at the RN. Without loss of generality, we further assume $g=1$ throughout this paper for notational convenience and thus $\mathbf{H}=\mathbf{H}_{2} \mathbf{P} \mathbf{H}_{1}$. Assume that the elements of $\mathbf{n}_{r}$ and $\mathbf{n}_{d}$ are identically and independently distributed (i.i.d.) complex Gaussian random variables with zero mean and variances $\sigma_{r}^{2}$ and $\sigma_{d}^{2}$, respectively, and then the correlation matrix of the overall noise vector, $\mathbf{n}$, is given by

$$
\mathbf{R}_{n}=E\left[\mathbf{n n}^{H}\right]=\sigma_{r}^{2} \mathbf{H}_{2} \mathbf{H}_{2}^{H}+\sigma_{d}^{2} \mathbf{I}_{N_{d}},
$$

where $E[\cdot]$ and $(\cdot)^{H}$ denote the expectation and the Hermitian transpose operators, respectively, and $\mathbf{I}_{N_{d}}$ denotes the $N_{d} \times N_{d}$ identity matrix.

In a practical two-hop MIMO AF relay system, it is attractive to keep minimum complexity at the RN that is supposed to be cost-effective. Thus it is desired that the RN does not undertake any task except simply amplifying and forwarding its received signals. In other words, no pilots are inserted at the $\mathrm{RN}$ to assist direct estimation of the forward relay channel at the DN. As a result, only the overall channel between the $\mathrm{SN}$ and the DN, H, can be estimated directly with the help of pilots inserted at the SN while the two cascaded relay channels over the SN-RN and the RN-DN hops, $\mathbf{H}_{1}$ and $\mathbf{H}_{2}$, remain unknown. However, Equation (2) indicates that the correlation matrix of the overall noise vector at the DN, based on which the maximum likelihood (ML) and the minimum mean-square error (MMSE) detection of a vertical bell laboratories layered space-time (V-BLAST) [16] system are performed, is determined by the forward relay channel over the RN-DN hop. Therefore, indirect estimation of the two cascaded relay channels at the DN of a two-hop MIMO AF system will effectively improve the system performance.

\section{B. Principle of Relay Channel Estimation at the DN}

Figure 2 shows an equivalent system for estimating cascaded relay channels at the DN. Our objective is to estimate the relay channels, $\mathbf{H}_{1}$ and $\mathbf{H}_{2}$, based on the predefined amplifying matrix at the RN, $\mathbf{P}$, and the corresponding overall channel, $\mathbf{H}=\mathbf{H}_{2} \mathbf{P H}_{1}$, which is assumed available at the DN through conventional channel estimation algorithms with the help of pilots inserted at the SN. Since the input $\mathbf{P}$ is known and 


\begin{tabular}{|l|l|l|l|}
\hline $\mathbf{H}_{o, 1}=\mathbf{H}_{2} \mathbf{P}_{1} \mathbf{H}_{1}$ & $\mathbf{H}_{o, 2}=\mathbf{H}_{2} \mathbf{P}_{2} \mathbf{H}_{1}$ & $\cdots$ & $\mathbf{H}_{o, L}=\mathbf{H}_{2} \mathbf{P}_{L} \mathbf{H}_{1}$ \\
\hline
\end{tabular}

Fig. 3. Principle of relay channel estimation

works like a pilot for relay channel estimation, it is called the pilot amplifying matrix in this paper. By varying the pilot amplifying matrix within several consecutive time slots, ${ }^{1}$ multiple equations with respect to (w.r.t.) $\mathbf{H}_{1}$ and $\mathbf{H}_{2}$ can be established and once the number of independent equations is large enough, relay channel estimation will be feasible.

The principle of relay channel estimation is further illustrated in Fig. 3 , in which $\mathbf{P}_{1}, \mathbf{P}_{2}, \cdots, \mathbf{P}_{L}$ denote the pilot amplifying matrices for $L$ consecutive time slots, and $\mathbf{H}_{o, 1}, \mathbf{H}_{o, 2}, \cdots, \mathbf{H}_{o, L}$ denote the corresponding overall channel matrices, i.e.,

$$
\mathbf{H}_{o, l}=\mathbf{H}_{2} \mathbf{P}_{l} \mathbf{H}_{1}, \quad 1 \leq l \leq L .
$$

Throughout this paper, we let $\mathbf{P}_{1}=\mathbf{I}_{N_{r}}$ since, from the perspective of relay channel estimation, the pilot amplifying matrix sequence, $\left[\mathbf{P}_{1}, \mathbf{P}_{2}, \cdots, \mathbf{P}_{L}\right]$, is equivalent to $\left[\mathbf{I}_{N_{r}}, \mathbf{P}_{1}^{-1} \mathbf{P}_{2}, \cdots, \mathbf{P}_{1}^{-1} \mathbf{P}_{L}\right]$. Obviously only $\alpha \mathbf{H}_{2}$ and $\frac{1}{\alpha} \mathbf{H}_{1}$, where $\alpha$ denotes an unknown complex scalar, can be obtained from (3) no matter how many different pilot amplifying matrices are utilized at the RN. However, since $\mathbf{H}_{2}$ (or $\mathbf{H}_{1}$ ) and $\alpha \mathbf{H}_{2}$ (or $\frac{1}{\alpha} \mathbf{H}_{1}$ ) share the same matrix structure, the knowledge of $\alpha \mathbf{H}_{2}$ and $\frac{1}{\alpha} \mathbf{H}_{1}$ at the DN can effectively improve the overall system performance, as will be demonstrated in Section VII.

Given the framework of relay channel estimation in Fig. 3, it is our interest to investigate what and how many pilot amplifying matrices are needed to guarantee feasible estimation of the two cascaded $\mathrm{MIMO}^{2}$ relay channels. In the rest of this paper, we will first assume perfect overall CSI at the DN and focus on the identifiability of the two relay channels for a given pilot amplifying matrix sequence. In particular, necessary and sufficient conditions for feasible relay channel estimation will be found and rules for designing low-complexity pilot amplifying matrices will be given accordingly.

\section{NeCESSARY AND SUfFicIENT CONDITIONS FOR Feasible Relay Channel Estimation}

In this section, we will investigate the identifiability of the two cascaded relay channels from matrix equation (3) for a given pilot amplifying matrix sequence. Since the equation w.r.t. $\mathbf{H}_{1}$ and $\mathbf{H}_{2}$ in (3) is nonlinear and difficult to analyze directly, we will first transform it into a linear one. For analytical convenience, we first consider a special case, $N_{r}=\min \left\{N_{s}, N_{d}\right\}$. The extension to the general case of $N_{r} \geq \min \left\{N_{s}, N_{d}\right\}$ will be discussed in Section VI.

\footnotetext{
${ }^{1}$ Throughout this paper, a time slot is defined as a period of time it takes to estimate the overall channel, $\mathbf{H}$. Furthermore, we assume slow fading channel so that the two relay channels remain constant during the whole estimation process.

${ }^{2}$ In the trivial case that either of the two relay channels is single-inputsingle-output (SISO), the pilot matrix at the RN reduces to a scalar and thus only one time slot is enough for feasible relay channel estimation.
}

\section{A. Transformation into a Linear Matrix Equation}

When $N_{r}=\min \left\{N_{s}, N_{d}\right\}$, transformation of (3) into a linear equation is straightforward. We first express $\mathbf{H}_{2}$ in terms of $\mathbf{H}_{1}$ or vice versa; then a linear matrix equation w.r.t. one of the two relay channels can be established by eliminating the other. To be brief, the original nonlinear matrix equation in (3) can be transformed into a linear one as ${ }^{3}$

$$
\mathbf{P}_{l} \mathbf{H}_{x}=\mathbf{H}_{x} \mathbf{Q}_{l}, \quad 2 \leq l \leq L,
$$

where

$$
\mathbf{H}_{x}= \begin{cases}\mathbf{H}_{1}, & \text { if } N_{r}=N_{s} \leq N_{d}, \\ \mathbf{H}_{2}^{-1}, & \text { if } N_{r}=N_{d} \leq N_{s},\end{cases}
$$

denotes the $N_{r} \times N_{r}$ channel matrix to estimate, and

$$
\mathbf{Q}_{l}= \begin{cases}\left(\mathbf{H}_{o, 1}^{H} \mathbf{H}_{o, 1}\right)^{-1} \mathbf{H}_{o, 1}^{H} \mathbf{H}_{o, l}, & \text { if } N_{r}=N_{s} \leq N_{d}, \\ \mathbf{H}_{o, l} \mathbf{H}_{o, 1}^{H}\left(\mathbf{H}_{o, 1} \mathbf{H}_{o, 1}^{H}\right)^{-1}, & \text { if } N_{r}=N_{d} \leq N_{s} .\end{cases}
$$

Given the linear matrix equation in (4), we need to estimate $\mathbf{H}_{x}$ based on the pilot matrix sequence, $\left\{\mathbf{P}_{l}, 2 \leq l \leq L\right\}$, and the corresponding transformed overall channel matrix sequence, $\left\{\mathbf{Q}_{l}, 2 \leq l \leq L\right\}$.

\section{B. First Necessary and Sufficient Condition}

The original matrix equation in (4) for relay channel estimation can be rewritten as [17]

$$
\left(\mathbf{I}_{N_{r}} \otimes \mathbf{P}_{l}-\mathbf{Q}_{l}^{T} \otimes \mathbf{I}_{N_{r}}\right) \cdot \mathbf{h}_{x}=0, \quad 2 \leq l \leq L,
$$

where $\mathbf{h}_{x}=\operatorname{vec}\left(\mathbf{H}_{x}\right)$ denotes the vectorization of $\mathbf{H}_{x}$ formed by stacking the columns of $\mathbf{H}_{x}$ into a single column vector, and $\otimes$ denotes the Kronecker product [17], [18].

Define

$$
\mathbf{A}=\left[\begin{array}{c}
\mathbf{A}_{2} \\
\mathbf{A}_{3} \\
\vdots \\
\mathbf{A}_{L}
\end{array}\right]=\left[\begin{array}{c}
\mathbf{I}_{N_{r}} \otimes \mathbf{P}_{2}-\mathbf{Q}_{2}^{T} \otimes \mathbf{I}_{N_{r}} \\
\mathbf{I}_{N_{r}} \otimes \mathbf{P}_{3}-\mathbf{Q}_{3}^{T} \otimes \mathbf{I}_{N_{r}} \\
\vdots \\
\mathbf{I}_{N_{r}} \otimes \mathbf{P}_{L}-\mathbf{Q}_{L}^{T} \otimes \mathbf{I}_{N_{r}}
\end{array}\right],
$$

which is an $(L-1) N_{r}^{2} \times N_{r}^{2}$ matrix, and then (7) can be rewritten in a compact manner as

$$
\mathbf{A} \cdot \mathbf{h}_{x}=0 .
$$

Since $\mathbf{h}_{x}$ is not a zero vector, $\mathbf{A}$ is not of full column rank, i.e., $\operatorname{rank}(\mathbf{A}) \leq N_{r}^{2}-1$. As a result, Equation (9) has an infinite number of solutions, including $\mathbf{h}_{x}$. In other words, the exact value of $\mathbf{h}_{x}$ cannot be determined no matter how many different pilot amplifying matrices are utilized at the RN, which coincides with our earlier analysis. For relay channel estimation, our objective is to obtain $\alpha \mathbf{h}_{x}$ from (9), where $\alpha$ denotes an unknown complex scalar, so as to learn about the matrix structure of $\mathbf{H}_{x}$. Therefore, the necessary and sufficient condition for feasible relay channel estimation is $\operatorname{rank}(\mathbf{A})=$ $N_{r}^{2}-1$, i.e., the solutions to (9) have only one degree of freedom (DoF) embodied in the unknown $\alpha$. Since $\mathbf{A}$ depends on not only the pilot amplifying matrix sequence, $\left\{\mathbf{P}_{l}, 2 \leq\right.$

\footnotetext{
${ }^{3}$ Note that we have assumed $L \geq 2$ since in the general case that both backward and forward relay channels are MIMO, relay channel estimation
} cannot be performed within only one time slot. 
$l \leq L\}$, but also the relay channels to estimate, $\mathbf{H}_{1}$ and $\mathbf{H}_{2}$, it is inconvenient to control the rank of $\mathbf{A}$ directly to meet this necessary and sufficient condition. To deal with this problem, we further define

$$
\mathbf{B}=\left[\begin{array}{c}
\mathbf{B}_{2} \\
\mathbf{B}_{3} \\
\vdots \\
\mathbf{B}_{L}
\end{array}\right]=\left[\begin{array}{c}
\mathbf{I}_{N_{r}} \otimes \mathbf{P}_{2}-\mathbf{P}_{2}^{T} \otimes \mathbf{I}_{N_{r}} \\
\mathbf{I}_{N_{r}} \otimes \mathbf{P}_{3}-\mathbf{P}_{3}^{T} \otimes \mathbf{I}_{N_{r}} \\
\vdots \\
\mathbf{I}_{N_{r}} \otimes \mathbf{P}_{L}-\mathbf{P}_{L}^{T} \otimes \mathbf{I}_{N_{r}}
\end{array}\right] .
$$

Similar to $\mathbf{A}, \mathbf{B}$ is also an $(L-1) N_{r}^{2} \times N_{r}^{2}$ matrix. Furthermore, we have shown in Appendix A that matrices $\mathbf{A}$ and $\mathbf{B}$ have the same $\operatorname{rank}$, i.e., $\operatorname{rank}(\mathbf{A})=\operatorname{rank}(\mathbf{B})$. Thus we obtain the following proposition.

Proposition 1. Given $\left\{\boldsymbol{P}_{l}, 2 \leq l \leq L\right\}$, the necessary and sufficient condition for feasible relay channel estimation is

$$
\operatorname{rank}(\boldsymbol{B})=N_{r}^{2}-1 .
$$

Different from A, B depends only on the pilot amplifying matrix sequence, independent of the relay channel to estimate. Therefore, given $\left\{\mathbf{P}_{l}, 2 \leq l \leq L\right\}$, it is convenient to decide on the identifiability of the two cascaded relay channels by checking the rank of $\mathbf{B}$.

Although Proposition 1 enables us to decide on the qualification of a given pilot amplifying matrix sequence, it does not provide any clue on rules to design qualified pilot matrices directly since it is still rather difficult to establish an explicit relationship between the pilot amplifying matrix sequence and the rank of $\mathbf{B}$. Therefore, we will define a new problem that is equivalent to the original problem of relay channel estimation. Based on the equivalent problem, rules will be developed conveniently to design qualified pilot amplifying matrices for relay channel estimation.

\section{Equivalent Problem and Second Necessary and Sufficient Condition}

Up to now, we have described the original problem of relay channel estimation, the definition of which is given below.

Original problem: Solve

$$
\mathbf{H}_{o, l}=\mathbf{H}_{2} \mathbf{P}_{l} \mathbf{H}_{1}, \quad 1 \leq l \leq L,
$$

to get $\mathbf{H}_{1}$ and $\mathbf{H}_{2}$.

To facilitate further analysis of the original problem, we define its equivalent problem as follows.

Equivalent problem: Solve

$$
\mathbf{P}_{l}=\mathbf{T}_{2} \mathbf{P}_{l} \mathbf{T}_{1}, \quad 1 \leq l \leq L,
$$

to get $N_{r} \times N_{r}$ square matrices $\mathbf{T}_{1}$ and $\mathbf{T}_{2}$.

It must be mentioned that both the original and the equivalent problems have an infinite number of solutions. ${ }^{4}$ In Appendix B, we have proved the following proposition.

Proposition 2. The solutions to the original problem and those to the equivalent problem have the same DoF.

\footnotetext{
${ }^{4}$ Note that $\mathbf{T}_{1}=\alpha \mathbf{I}_{N_{r}}$ and $\mathbf{T}_{2}=\frac{1}{\alpha} \mathbf{I}_{N_{r}}$, where $\alpha$ denotes an arbitrary complex scalar, are always solutions to the equivalent problem.
}

The equivalence between the two problems indicated in Proposition 2 significantly facilitates analysis of the identifiability of the two relay channels for a given pilot amplifying matrix sequence. As mentioned, the necessary and sufficient condition for feasible relay channel estimation is that the solutions to the original problem have only one DoF. Thus, from Proposition 2, we obtain the second necessary and sufficient condition for feasible relay channel estimation as follows.

Proposition 3. Given $\left\{\boldsymbol{P}_{l}, 2 \leq l \leq L\right\}$, relay channel estimation is feasible if and only if $\boldsymbol{T}_{1}=\alpha \boldsymbol{I}_{N_{r}}$ and $\boldsymbol{T}_{2}=\frac{1}{\alpha} \boldsymbol{I}_{N_{r}}$, where $\alpha$ denotes an arbitrary complex scalar, are the sole solutions to the equivalent problem.

In practice, the $\mathrm{RN}$ in the two-hop MIMO AF relay system needs to maintain a minimum complexity. Therefore, it is attractive to set the pilot amplifying matrices as the permutations of an identity matrix since, in this case, the matrix multiplication manipulation at the $\mathrm{RN}$ can be realized with minimum complexity by switching the connection between the receive and the transmit antennas of the RN. Unfortunately, with the help of Proposition 3, we have proved in Appendix $\mathrm{C}$ that it is impossible to estimate the two cascaded relay channels at the DN with such pilot amplifying matrices.

\section{Third Necessary and Sufficient Condition}

In light of Proposition 3, we will reinvestigate the identifiability of the two relay channels for a given pilot amplifying matrix sequence by examining the DoF of the solutions to the equivalent problem.

Since $\mathbf{P}_{1}=\mathbf{T}_{2} \mathbf{P}_{1} \mathbf{T}_{1}$ and $\mathbf{P}_{1}=\mathbf{I}_{N_{r}}$, we have $\mathbf{T}_{1}=\mathbf{T}_{2}^{-1}$. Thus (13) can be rewritten as

$$
\mathbf{T}_{2}=\mathbf{P}_{l} \mathbf{T}_{2} \mathbf{P}_{l}^{-1}, \quad 2 \leq l \leq L .
$$

Perform eigenvalue decomposition on $\mathbf{T}_{2}$ to get $\mathbf{T}_{2}=$ $\mathbf{U} \boldsymbol{\Lambda} \mathbf{U}^{-1}, 5$ where $\mathbf{U}$ and $\boldsymbol{\Lambda}$ denote the eigenvector and the eigenvalue matrices of $\mathbf{T}_{2}$, respectively. Then (14) can be rewritten as

$$
\mathbf{T}_{2}=\mathbf{P}_{l} \mathbf{U} \cdot \boldsymbol{\Lambda} \cdot\left(\mathbf{P}_{l} \mathbf{U}\right)^{-1}, \quad 2 \leq l \leq L,
$$

which indicates that, for any $2 \leq l \leq L, \mathbf{P}_{l} \mathbf{U}$ is also an eigenvector matrix of $\mathbf{T}_{2}$.

According to Proposition 3, relay channel estimation is feasible if and only if $\mathbf{T}_{2}$ in (15) has only one DoF. To find the necessary and sufficient condition for that, we first perform eigenvalue decomposition on each pilot matrix to get

$$
\mathbf{P}_{l}=\mathbf{V}_{l} \boldsymbol{\Lambda}_{l} \mathbf{V}_{l}^{-1}, \quad 2 \leq l \leq L,
$$

where $\mathbf{V}_{l}=\left[\mathbf{v}_{l 1}, \mathbf{v}_{l 2}, \cdots, \mathbf{v}_{l N_{r}}\right]$ and $\mathbf{v}_{l n}, 1 \leq n \leq N_{r}$, denotes the $n$th eigenvector of $\mathbf{P}_{l}$. Then we group the $N_{r}$ eigenvectors of $\mathbf{P}_{l}$ into $K$ clusters with $n_{k}$ eigenvectors in the $k$ th cluster $V_{l k}, 1 \leq k \leq K$, such that $V_{l k}$ 's with $2 \leq l \leq L$ span the same $n_{k}$-dimensional space $S_{k}$. In particular, such eigenvector grouping is performed over $\left\{\mathbf{P}_{l}, 2 \leq l \leq L\right\}$ so that the number of their eigenvector clusters, $K$, is as large

\footnotetext{
${ }^{5}$ Since $\mathbf{P}_{l}$ is unitary and $\mathbf{P}_{l}=\mathbf{T}_{2} \mathbf{P}_{l} \mathbf{T}_{1}, \mathbf{T}_{2}$ and $\mathbf{T}_{1}$ must be nonsingular.
} 
as possible. In the case that $\mathbf{P}_{l}$ has an $n$-dimensional $(n>1)$ eigenspace associated with an $n$-order repeated eigenvalue, $n$ eigenvectors are chosen appropriately from this eigenspace for grouping so that $K$ is maximized. Given the above eigenvector grouping rule, we have proved the following third necessary and sufficient condition for feasible relay channel estimation in Appendix D.

Proposition 4. Given $\left\{\boldsymbol{P}_{l}, 2 \leq l \leq L\right\}$, relay channel estimation is feasible if and only if they have only one eigenvector cluster, i.e., $K=1$.

The third necessary and sufficient condition given by Proposition 4 can be used not only to determine whether a given pilot matrix sequence ensures feasible relay channel estimation, but also to design qualified pilot matrix sequence directly. According to Proposition 4, it is impossible to estimate the two MIMO relay channels within two time slots since, in this case, $L=2$ and the number of eigenvector clusters of $\mathbf{P}_{2}$ alone is $N_{r}$, which is greater than one. On the other hand, for appropriately designed $\mathbf{P}_{2}$ and $\mathbf{P}_{3}$, the number of their eigenvector clusters can be as small as one. In other words, the minimum number of time slots required for feasible relay channel estimation is 3, i.e., $L_{\min }=3$. In Section IV, rules will be developed to design low-complexity $\mathbf{P}_{2}$ and $\mathbf{P}_{3}$ meeting the third necessary and sufficient condition such that the two relay channels can be estimated within three time slots.

\section{DESIGN Rules}

In this section, we develop rules to design low-complexity pilot amplifying matrices so that the relay channels can be estimated with minimum complexity at the RN. According to Proposition 4, we need to ensure that the number of eigenvector clusters of the designed pilot matrices is one.

As mentioned, it is always attractive to use a diagonal matrix or a quasi-diagonal matrix (a permutation of a diagonal matrix) as the pilot amplifying matrix at the $\mathrm{RN}$ so as to reduce the complexity in the matrix multiplication manipulation. Before giving the specific design rules, we first present the following definitions regarding a permutation operation.

Denote $\mathbf{D}$ and $\mathbf{F}$ as two $N_{r} \times N_{r}$ square matrices. Let $\mathbf{F}$ be a permutation of $\mathbf{D}$, i.e., $\mathbf{F}$ is obtained by switching the row order of $\mathbf{D}$, and then we define

- If the $i$ th row of $\mathbf{F}$ comes from the $j$ th row of $\mathbf{D}, i$ is directly reachable from $j, 1 \leq i, j \leq N_{r}$, which is denoted as $f(j)=i$ and $f^{-1}(i)=j$. $^{6}$

- If $i$ is directly reachable from $j$ and $j$ is directly reachable from $k, i$ is reachable from $k$.

- A permutation is indivisible if any $i$ and $j\left(1 \leq i, j \leq N_{r}\right)$ reach each other.

Given the above definitions, we present the following design rules.

- Let $\mathbf{P}_{1}=\mathbf{I}_{N_{r}}$.

- Generate $N_{r}$ distinct complex numbers, each with unit magnitude, to get a diagonal $\mathbf{P}_{2}$.

- Let $\mathbf{P}_{3}$ be an indivisible permutation of $\mathbf{P}_{2}$.

${ }^{6}$ Note that, for any permutation, there exists a unique $j$ that directly reaches $i$ and a unique $k$ that is directly reachable from $i$.
With the above design rules, we have shown in Appendix $\mathrm{E}$ that $\mathbf{P}_{2}$ and $\mathbf{P}_{3}$ have only one eigenvector cluster and thus the two cascaded relay channels can be estimated within three time slots. Among all of the $N_{r}$ ! permutations of $\mathbf{P}_{2}$, there are $\left(N_{r}-1\right)$ ! indivisible ones that qualify as $\mathbf{P}_{3}$ candidates. In practice, $\mathbf{P}_{3}$ can be obtained by simply cyclically shifting the rows of $\mathbf{P}_{2}$. To illustrate the above design rules, we give a pilot amplifying matrix sequence candidate for relay channel estimation in a two-hop MIMO AF relay system with $N_{r}=2$ as follows.

$$
\mathbf{P}_{1}=\left[\begin{array}{ll}
1 & 0 \\
0 & 1
\end{array}\right], \mathbf{P}_{2}=\left[\begin{array}{cc}
1 & 0 \\
0 & -1
\end{array}\right], \mathbf{P}_{3}=\left[\begin{array}{cc}
0 & 1 \\
-1 & 0
\end{array}\right] .
$$

The above design rules are especially attractive in practice because, firstly, the two relay channels can be estimated within the minimum three time slots, secondly, application of diagonal and quasi-diagonal pilot amplifying matrices maintains a minimum complexity at the RN, and thirdly, the orthogonality of these pilot amplifying matrices ensures a constant power amplifying gain at the RN.

\section{LINEAR LEAST-SQUARE ESTIMATION OF RELAY CHANNELS IN A NOISY ENVIRONMENT}

So far, we have assumed perfect overall CSI at the DN and focused on the design of the pilot matrix sequence to ensure feasible relay channel estimation. In this section, we will consider imperfect overall CSI at the DN and develop a specific algorithm to estimate the relay channels in such a noisy environment.

In practice, the overall channel between the $\mathrm{SN}$ and the $\mathrm{DN}$, $\mathbf{H}_{o, l}$, has to be estimated at the DN. The estimated overall channel is always corrupted by noise and can be expressed as

$$
\mathbf{H}_{o, l}^{N}=\mathbf{H}_{o, l}+\mathbf{N}_{o, l}, \quad 1 \leq l \leq L,
$$

where $\mathbf{H}_{o, l}$ is the actual overall channel and $\mathbf{N}_{o, l}$ denotes the additive estimation noise matrix. Accordingly, the corrupted version of $\mathbf{Q}_{l}$ in (6) is given by

$$
\mathbf{Q}_{l}^{N}=\left\{\begin{array}{c}
\left(\left(\mathbf{H}_{o, 1}^{N}\right)^{H} \mathbf{H}_{o, 1}^{N}\right)^{-1}\left(\mathbf{H}_{o, 1}^{N}\right)^{H} \mathbf{H}_{o, l}^{N}, \text { if } N_{r}=N_{s} \leq N_{d}, \\
\mathbf{H}_{o, l}^{N}\left(\mathbf{H}_{o, 1}^{N}\right)^{H}\left(\mathbf{H}_{o, 1}^{N}\left(\mathbf{H}_{o, 1}^{N}\right)^{H}\right)^{-1}, \text { if } N_{r}=N_{d} \leq N_{s}
\end{array}\right.
$$

which can be equivalently rewritten as $\mathbf{Q}_{l}^{N}=\mathbf{Q}_{l}+\mathbf{N}_{Q, l}$ where $\mathbf{N}_{Q, l}$ denotes an equivalent additive noise matrix. As a result, the original linear matrix equation in (4) is revised as

$$
\mathbf{P}_{l} \mathbf{H}_{x}-\mathbf{H}_{x} \mathbf{Q}_{l}^{N}+\mathbf{H}_{x} \mathbf{N}_{Q, l}=0, \quad 2 \leq l \leq L
$$

Define

$$
\mathbf{A}_{N}=\left[\begin{array}{c}
\mathbf{A}_{2}^{N} \\
\mathbf{A}_{3}^{N} \\
\vdots \\
\mathbf{A}_{L}^{N}
\end{array}\right]=\left[\begin{array}{c}
\mathbf{I}_{N_{r}} \otimes \mathbf{P}_{2}-\left(\mathbf{Q}_{2}^{N}\right)^{T} \otimes \mathbf{I}_{N_{r}} \\
\mathbf{I}_{N_{r}} \otimes \mathbf{P}_{3}-\left(\mathbf{Q}_{3}^{N}\right)^{T} \otimes \mathbf{I}_{N_{r}} \\
\vdots \\
\mathbf{I}_{N_{r}} \otimes \mathbf{P}_{L}-\left(\mathbf{Q}_{L}^{N}\right)^{T} \otimes \mathbf{I}_{N_{r}}
\end{array}\right]
$$


and

$$
\mathbf{n}_{H_{x}}=\left[\begin{array}{c}
\operatorname{vec}\left(\mathbf{H}_{x} \mathbf{N}_{Q, 2}\right) \\
\operatorname{vec}\left(\mathbf{H}_{x} \mathbf{N}_{Q, 3}\right) \\
\vdots \\
\operatorname{vec}\left(\mathbf{H}_{x} \mathbf{N}_{Q, L}\right)
\end{array}\right],
$$

and then (20) can be rewritten as

$$
\mathbf{A}_{N} \cdot \mathbf{h}_{x}+\mathbf{n}_{H_{x}}=0
$$

where $\mathbf{h}_{x}=\operatorname{vec}\left(\mathbf{H}_{x}\right)=\left[h_{x, 1}, h_{x, 2}, \cdots, h_{x, N_{r}^{2}}\right]^{T}$ denotes the vectorization of $\mathbf{H}_{x}$. As mentioned, we can only obtain $\alpha \mathbf{h}_{x}$, where $\alpha$ denotes an unknown complex scalar, through relay channel estimation. Considering this, we try to estimate the value of $\mathbf{h}_{x}$ normalized to $h_{x, 1}$, namely $\mathbf{h}_{x, n}=\frac{1}{h_{x, 1}} \mathbf{h}_{x}$, by adding an extra equation to (23) to get

$$
\left[\begin{array}{c}
\mathbf{A}_{N} \\
\mathbf{e}
\end{array}\right] \cdot \mathbf{h}_{x, n}+\left[\begin{array}{c}
\mathbf{n}_{H_{x}} \\
0
\end{array}\right]=\mathbf{y}
$$

where $\mathbf{e}=[1,0,0, \cdots, 0]$ is an $N_{r}^{2}$-dimensional row vector and $\mathbf{y}=[0,0, \cdots, 1]^{T}$ is an $(L-1) N_{r}^{2}+1$-dimensional column vector. Thus $\mathbf{h}_{x, n}$ can be estimated as an approximate LS solution [17] to (24) as ${ }^{7}$

$$
\hat{\mathbf{h}}_{x, n, L S}=\left(\mathbf{C}^{H} \mathbf{C}\right)^{-1} \mathbf{C}^{H} \mathbf{y},
$$

where $\mathbf{C}=\left[\begin{array}{ll}\mathbf{A}_{N}^{T} & \mathbf{e}^{T}\end{array}\right]^{T}$. According to (24), the estimation error of $\hat{\mathbf{h}}_{x, n, L S}$ is given by

$$
\hat{\mathbf{h}}_{x, n, L S}-\mathbf{h}_{x, n}=\left(\mathbf{C}^{H} \mathbf{C}\right)^{-1} \mathbf{C}^{H} \tilde{\mathbf{n}}_{H_{x}},
$$

where $\tilde{\mathbf{n}}_{H_{x}}=\left[\begin{array}{ll}\mathbf{n}_{H_{x}}^{T} & 0\end{array}\right]^{T}$. Accordingly, the mean-square error (MSE) of $\hat{\mathbf{h}}_{x, n, L S}$ can be expressed as

$$
\xi=E\left[\left(\hat{\mathbf{h}}_{x, n, L S}-\mathbf{h}_{x, n}\right)^{H}\left(\hat{\mathbf{h}}_{x, n, L S}-\mathbf{h}_{x, n}\right)\right],
$$

where $E[\cdot]$ denotes expectation w.r.t. both the random noise and the relay channel to estimate. Since both $\mathbf{C}$ and $\tilde{\mathbf{n}}_{H_{x}}$ depend on not only the random noise but also the relay channel to estimate, it is rather difficult to obtain a closedform expression for $\xi$. Therefore, it can only be evaluated numerically. While the focus of this paper is on investigating the feasibility of estimating the cascaded relay channels at the DN and designing low-complexity pilot amplifying matrices at the $\mathrm{RN}$, it will be a potential future research topic to design the optimal pilot matrices to minimize the MSE in a noisy environment.

\section{Extension to General CASE}

In order for the overall channel to be nonsingular, we have assumed $N_{r} \geq \min \left\{N_{s}, N_{d}\right\}$, which consists of Case I: $N_{r}=\min \left\{N_{s}, N_{d}\right\}$ and Case II: $N_{r}>\min \left\{N_{s}, N_{d}\right\}$.

\footnotetext{
${ }^{7}$ Since the noise part, $\mathbf{n}_{H_{x}}$, depends on the unknown channel $\mathbf{H}_{x}, \hat{\mathbf{h}}_{x, n, L S}$ is not an exact LS solution to (24). In the presence of imperfect overall CSI, Equation (24) turns out to be a nonlinear one w.r.t $\mathbf{h}_{x, n}$ and is hard to solve. When a relatively accurate overall channel is available, i.e., $\mathbf{Q}_{l}^{N} \approx \mathbf{Q}_{l}$, the power of $\mathbf{n}_{H_{x}}$ is low. In this case, $\hat{\mathbf{h}}_{x, n, L S}$ is an approximate linear LS solution to (24).
}

In the previous sections, we have investigated relay channel estimation at the DN in Case I; in this section, we will extend it to Case II.

In Case II, the original nonlinear matrix equation in (3) cannot be transformed into a linear one w.r.t either one of the two cascaded relay channels. Therefore, we propose to estimate the relay channels via multiple steps. In each step, the relay channel estimation procedure in Case I is applied. Specifically, we divide the antennas at the RN into multiple groups so that the number of antennas in each group, $\tilde{N}_{r}$, equals $\min \left\{N_{s}, N_{d}\right\}$. Obviously there will be overlapping antennas in different groups if $I=\frac{N_{r}}{\tilde{N}_{r}}$ is not an integer. Without loss of generality, we assume $I$ is an integer to facilitate explanations. By grouping the antennas at the RN, the original two-hop MIMO AF relay system is decomposed into $I$ parallel subsystems with still $N_{s}$ and $N_{d}$ antennas at the SN and the DN, respectively, but $\tilde{N}_{r}$ antennas at the RN. Let $\mathbf{H}_{1, i}$ and $\mathbf{H}_{2, i}, 1 \leq i \leq I$, denote the backward and the forward relay channels of the $i$ th subsystem, respectively, and then the overall relay channels are given by

$$
\mathbf{H}_{1}=\left[\mathbf{H}_{1,1}^{T}, \mathbf{H}_{1,2}^{T}, \cdots, \mathbf{H}_{1, I}^{T}\right]^{T},
$$

and

$$
\mathbf{H}_{2}=\left[\mathbf{H}_{2,1}, \mathbf{H}_{2,2}, \cdots, \mathbf{H}_{2, I}\right] .
$$

Since $\tilde{N}_{r}=\min \left\{N_{s}, N_{d}\right\}, \mathbf{H}_{1, i}$ and $\mathbf{H}_{2, i}$ can be estimated following the procedure in Case I by switching off all of the antennas of the RN that are outside the $i$ th group. Then the overall relay channels, $\mathbf{H}_{1}$ and $\mathbf{H}_{2}$, can be obtained by combining $\mathbf{H}_{1, i}$ 's and $\mathbf{H}_{2, i}$ 's, respectively. Since we can only get the values of $\mathbf{H}_{1, i}$ and $\mathbf{H}_{2, i}$ normalized to one of their respective elements, a number of extra antenna groups are needed to obtain the amplitude and phase relationship between the relay channels of different subsystems. To illustrate the relay channel estimation procedure in Case II, we take a 2/4/4 $\left(N_{s}=2, N_{r}=4\right.$, and $\left.N_{d}=4\right)$ two-hop MIMO AF relay system as an example. The four antennas at the RN, $a_{1}, a_{2}, a_{3}$, and $a_{4}$, are divided into two $(I=2)$ groups. For example, $a_{1}$ and $a_{2}$ form Group 1, and $a_{3}$ and $a_{4}$ form Group 2. Thus the original $2 / 4 / 4$ system is decomposed into two $2 / 2 / 4$ subsystems. To determine the amplitude and phase relationship between the relay channels of the two subsystems, we further let $a_{1}$ and $a_{3}$ form Group 3 to get another $2 / 2 / 4$ subsystem.

\section{Simulation Results}

In this section, we present some simulation results to demonstrate the performance of the approximate linear LS estimation of the relay channels developed in Section V. In our simulation, a 4/4/4 $\left(N_{s}=N_{r}=N_{d}=4\right)$ two-hop MIMO AF relay system is considered. The elements of $\mathbf{H}_{1}, \mathbf{H}_{2}, \mathbf{n}_{r}$, and $\mathbf{n}_{d}$ are all modeled as i.i.d. complex Gaussian random variables with zero mean and unit variance. Furthermore, the power amplifying gain of the RN is set to one, i.e., $g=1$. Denote $P_{s}$ as the transmit power of each antenna at the SN, the value of which is also defined as the system SNR of the overall system. Three pilot amplifying matrices are generated randomly for relay channel estimation according to the design rules given in Section IV. The normalized relay channels, 
$\overline{\mathbf{H}}_{1}=\frac{1}{\mathbf{H}_{1}(1,1)} \mathbf{H}_{1}$ and $\overline{\mathbf{H}}_{2}=\frac{1}{\mathbf{H}_{2}(1,1)} \mathbf{H}_{2}$, where $\mathbf{H}_{i}(1,1)$ denotes the element at the first row and the first column of $\mathbf{H}_{i}, 1 \leq i \leq 2$, are estimated through the approximate linear LS estimation procedure developed in Section V. The corresponding normalized MSE of estimate $\overline{\mathbf{H}}_{i, E}$ is defined as

$$
\xi_{i}=E\left[\frac{\left\|\overline{\mathbf{H}}_{i}-\overline{\mathbf{H}}_{i, E}\right\|^{2}}{\left\|\overline{\mathbf{H}}_{i}\right\|^{2}}\right], \quad 1 \leq i \leq 2,
$$

where $\|\cdot\|^{2}$ denotes the sum power of all elements of a matrix.

Figure 4 shows the normalized MSE curves of the estimated relay channels, $\overline{\mathbf{H}}_{1, E}$ and $\overline{\mathbf{H}}_{2, E}$. In Fig. 4(a), we apply the overall channel model with additive noise given in (18). In particular, we assume that elements of the additive noise matrix are i.i.d. Gaussian with zero mean and variance $\sigma_{n}^{2}$ and define the equivalent SNR as the ratio of the average power of the overall channel to that of the additive noise, i.e.,

$$
\gamma_{e q}=\frac{E\left[\left\|\mathbf{H}_{o, l}\right\|^{2}\right]}{N_{s} N_{d} \sigma_{n}^{2}}=\frac{N_{r}}{\sigma_{n}^{2}} .
$$

In contrast, the imperfect overall channel in Fig. 4(b) is obtained by employing the linear MMSE (LMMSE) channel estimation algorithm under a certain system SNR. Figure 4 indicates that an acceptable estimation performance, such as a normalized MSE smaller than $-5 \mathrm{~dB}$, can be achieved when the equivalent SNR of the overall channel (in Fig. 4(a)), or the system SNR (in Fig. 4(b)), is greater than 15 $\mathrm{dB}$. This indicates that the accuracy of the overall channel is significant to relay channel estimation. While $\overline{\mathbf{H}}_{1, E}$ and $\overline{\mathbf{H}}_{2, E}$ have the same normalized MSE under the overall channel model with additive white Gaussian noise, it is interesting to notice that $\overline{\mathbf{H}}_{1, E}$ has a smaller normalized MSE than $\overline{\mathbf{H}}_{2, E}$ when the overall channel is obtained through the LMMSE channel estimation algorithm. This is actually reasonable since the overall system model in (1) indicates that $\mathbf{H}_{1}$ and $\mathbf{H}_{2}$ are not playing interchangeable roles in the original system. Specifically, $\mathbf{H}_{1}$ is independent of the overall noise at the DN while $\mathbf{H}_{2}$ is not since it conveys $\mathbf{n}_{r}$, the noise vector at the $\mathrm{RN}$, to the DN. While the focus of this paper is on pilot matrix design for estimating cascaded relay channels based on available overall CSI, it will be a potential future research topic to jointly estimate $\mathbf{H}_{1}, \mathbf{H}_{2}$, and the overall channel.

Figure 5 demonstrates the performance improvement of the two-hop MIMO AF relay system when the estimated forward relay channel is utilized to improve signal detection at the DN. In our simulation, V-BLAST transmission with QPSK modulation is applied at the $\mathrm{SN}$ and no error correcting coding is utilized. Imperfect overall channel is obtained through the LMMSE channel estimation algorithm and, based on that, the ML and MMSE detection schemes are applied at the DN. In order to get accurate overall channel estimation for system performance optimization, we assume that the SNR of the pilot for overall channel estimation is $10 \mathrm{~dB}$ higher than that of the data. In particular, we assume that the average power gain over the RN-DN hop, $\sigma_{2}^{2}$, which equals one in our simulation, is known at the DN since it is static and thus can be easily obtained. When relay CSI over the RN-DN hop is unavailable, we simply assume that the overall noise is white and estimate

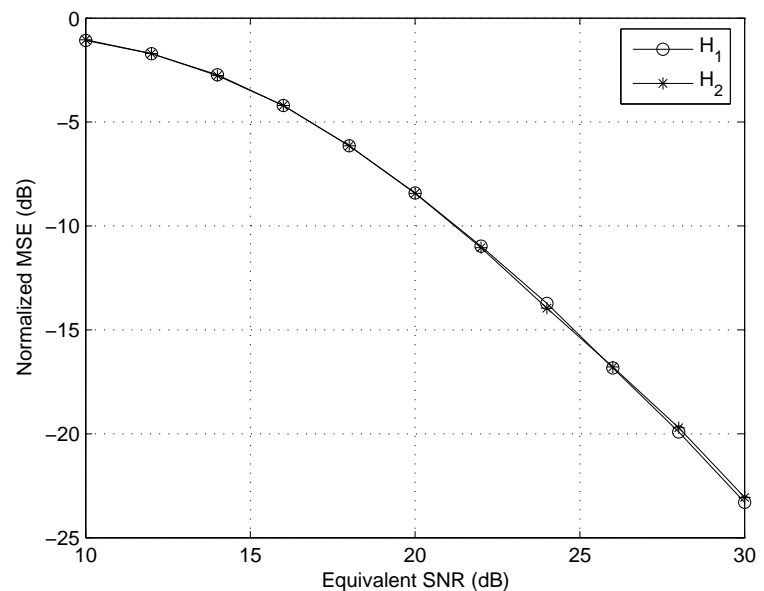

(a) Normalized MSE versus equivalent SNR of the overall channel

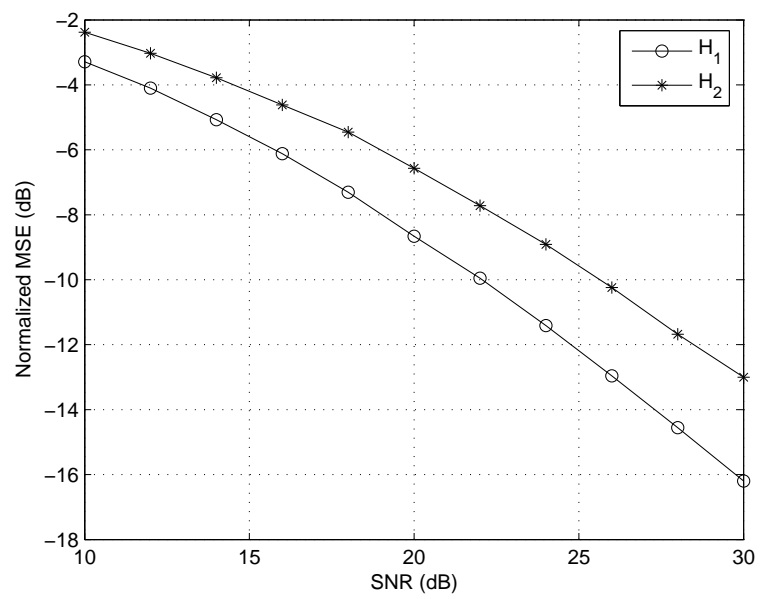

(b) Normalized MSE versus system SNR

Fig. 4. Normalized MSE curves of the estimated relay channels

its correlation matrix as

$$
\tilde{\mathbf{R}}_{n}=\sigma_{r}^{2} E\left[\mathbf{H}_{2} \mathbf{H}_{2}^{H}\right]+\sigma_{d}^{2} \mathbf{I}_{N_{d}}=\left(N_{r} \sigma_{r}^{2} \sigma_{2}^{2}+\sigma_{d}^{2}\right) \mathbf{I}_{N_{d}},
$$

based on which approximate ML and MMSE detection schemes are applied at the DN. In contrast, with relay channel estimation at the $\mathrm{DN}$, we estimate the correlation matrix of the overall noise vector as

$$
\hat{\mathbf{R}}_{n}=\sigma_{r}^{2} \frac{N_{d} N_{r} \sigma_{2}^{2}}{\left\|\overline{\mathbf{H}}_{2, E}\right\|^{2}} \overline{\mathbf{H}}_{2, E} \overline{\mathbf{H}}_{2, E}^{H}+\sigma_{d}^{2} \mathbf{I}_{N_{d}},
$$

based on which improved approximate ML and MMSE detection schemes can be applied at the DN. From Fig. 5, we observe that when BER is around $10^{-3}$, the ML and the MMSE detection schemes based on the estimated relay channels achieve about $1.0 \mathrm{~dB}$ performance gains over those based on the white overall noise assumption, respectively. While the initial simulation results have justified estimating the cascaded relay channels at the DN, further utilization of the estimated relay channels for system performance improvement will be a future research topic. 


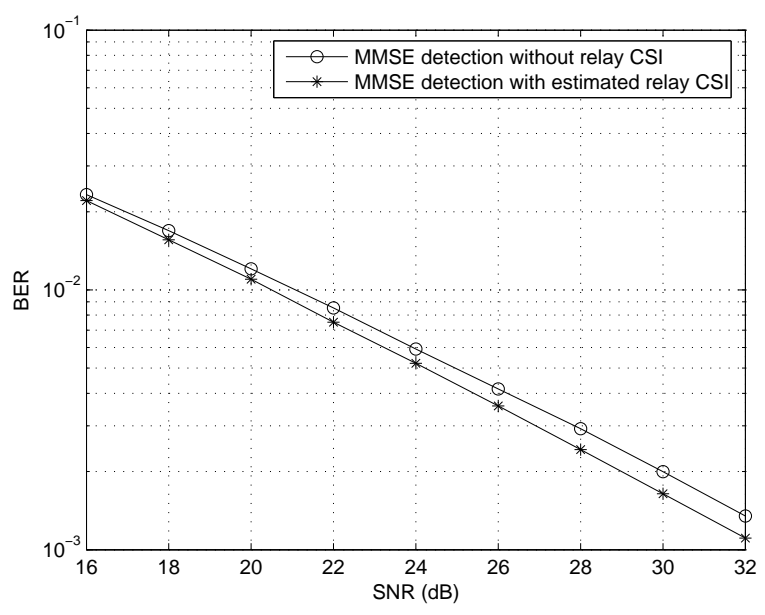

(a) MMSE detection

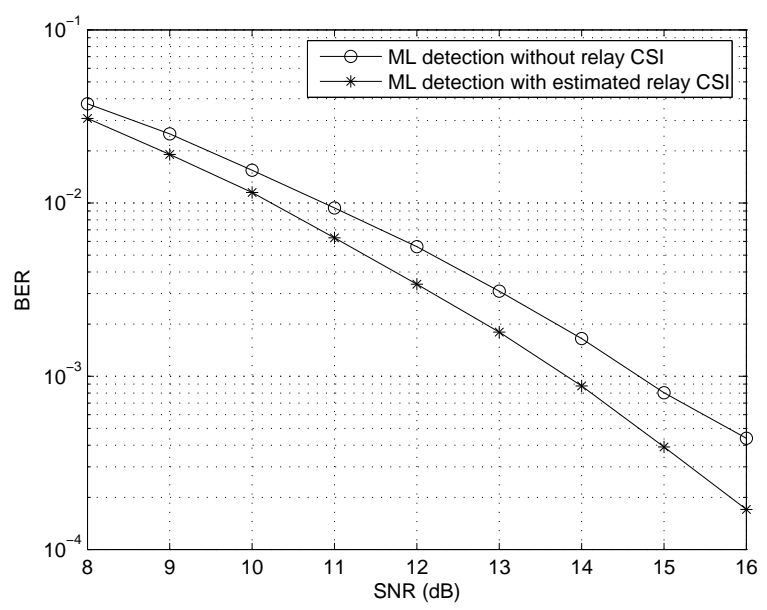

(b) ML detection

Fig. 5. BER performance improvement achieved by relay channel estimation

\section{CONCLUSION}

In this paper, we have investigated estimating the cascaded backward and forward relay channels at the final destination of a two-hop MIMO AF relay system based on a predefined pilot amplifying matrix sequence at the $\mathrm{RN}$ and the corresponding overall channel sequence obtained at the DN through conventional channel estimation algorithms. Such a relay channel estimation scheme is applicable to a minimum-complexity relay that simply amplifies and forwards its received signals. We have found the necessary and sufficient conditions on the pilot matrix sequence to ensure feasible relay channel estimation and presented rules to design diagonal or quasidiagonal pilot amplifying matrices to meet these conditions. In the presence of imperfect overall CSI at the DN, we have further developed the approximate linear LS estimation of the relay channels and evaluated its performance by simulation results. Furthermore, initial simulation results have also demonstrated the system performance improvement achieved by relay channel estimation.

\section{APPENDIX A}

According to (4), $\mathbf{Q}_{l}=\mathbf{H}_{x}^{-1} \mathbf{P}_{l} \mathbf{H}_{x}, 2 \leq l \leq L$, where $\mathbf{H}_{x}$ denotes the $N_{r} \times N_{r}$ relay channel matrix to estimate. Thus, according to the mixed-product property of the Kronecker product [17], we have

$$
\begin{aligned}
\mathbf{A}_{l}= & \mathbf{I} \otimes \mathbf{P}_{l}-\mathbf{Q}_{l}^{T} \otimes \mathbf{I} \\
= & \mathbf{H}_{x}^{T}\left(\mathbf{H}_{x}^{T}\right)^{-1} \otimes \mathbf{P}_{l}-\mathbf{H}_{x}^{T} \mathbf{P}_{l}^{T}\left(\mathbf{H}_{x}^{T}\right)^{-1} \otimes \mathbf{I} \\
= & \left(\mathbf{H}_{x}^{T} \otimes \mathbf{I}\right)\left[\left(\mathbf{H}_{x}^{T}\right)^{-1} \otimes \mathbf{P}_{l}\right]-\left(\mathbf{H}_{x}^{T} \otimes \mathbf{I}\right)\left[\mathbf{P}_{l}^{T}\left(\mathbf{H}_{x}^{T}\right)^{-1} \otimes \mathbf{I}\right] \\
= & \left(\mathbf{H}_{x}^{T} \otimes \mathbf{I}\right)\left(\mathbf{I} \otimes \mathbf{P}_{l}\right)\left[\left(\mathbf{H}_{x}^{T}\right)^{-1} \otimes \mathbf{I}\right] \\
& -\left(\mathbf{H}_{x}^{T} \otimes \mathbf{I}\right)\left(\mathbf{P}_{l}^{T} \otimes \mathbf{I}\right)\left[\left(\mathbf{H}_{x}^{T}\right)^{-1} \otimes \mathbf{I}\right] \\
= & \left(\mathbf{H}_{x}^{T} \otimes \mathbf{I}\right)\left(\mathbf{I} \otimes \mathbf{P}_{l}-\mathbf{P}_{l}^{T} \otimes \mathbf{I}\right)\left(\mathbf{H}_{x}^{T} \otimes \mathbf{I}\right)^{-1},
\end{aligned}
$$

where $\mathbf{I}$ denotes the $N_{r} \times N_{r}$ identity matrix. Define $\mathbf{W}=$ $\mathbf{H}_{x}^{T} \otimes \mathbf{I}$; then, according to (A-1) and (10), $\mathbf{A}_{l}=\mathbf{W B}_{l} \mathbf{W}^{-1}$, and therefore

$$
\mathbf{A}=\left[\begin{array}{c}
\mathbf{A}_{2} \\
\mathbf{A}_{3} \\
\vdots \\
\mathbf{A}_{L}
\end{array}\right]=\left[\begin{array}{cccc}
\mathbf{W} & 0 & \cdots & 0 \\
0 & \mathbf{W} & \cdots & 0 \\
\vdots & \vdots & \ddots & \vdots \\
0 & 0 & \cdots & \mathbf{W}
\end{array}\right]\left[\begin{array}{c}
\mathbf{B}_{2} \\
\mathbf{B}_{3} \\
\vdots \\
\mathbf{B}_{L}
\end{array}\right] \mathbf{W}^{-1}
$$

Since $\mathbf{W}$ is invertible, (A-2) verifies that $\operatorname{rank}(\mathbf{A})=\operatorname{rank}(\mathbf{B})$.

\section{APPENDIX B}

For the equivalent problem, the original matrix equation w.r.t. $\mathbf{T}_{1}$ and $\mathbf{T}_{2}$ is given by

$$
\mathbf{P}_{l}=\mathbf{T}_{2} \mathbf{P}_{l} \mathbf{T}_{1}, \quad 1 \leq l \leq L,
$$

Since $\mathbf{P}_{1}=\mathbf{I}_{N_{r}}$ and $\mathbf{P}_{1}=\mathbf{T}_{2} \mathbf{P}_{1} \mathbf{T}_{1}, \mathbf{T}_{2}=\mathbf{T}_{1}^{-1}$. Therefore, (A-3) can be rewritten as

$$
\mathbf{P}_{l} \mathbf{T}_{1}=\mathbf{T}_{1} \mathbf{P}_{l}, \quad 2 \leq l \leq L,
$$

Similar to (9), (A-4) can be rewritten in a compact manner as follows

$$
\mathbf{B} \cdot \mathbf{t}_{1}=0 .
$$

where $\mathbf{B}$ is an $(L-1) N_{r}^{2} \times N_{r}^{2}$ matrix defined in (10) and $\mathbf{t}_{1}=\operatorname{vec}\left(\mathbf{T}_{1}\right)$ denotes the vectorization of $\mathbf{T}_{1}$. Obviously the solutions to (A-5) have $N_{r}^{2}-\operatorname{rank}(\mathbf{B})$ DoF's. According to (9), the solutions to the original problem have $N_{r}^{2}-\operatorname{rank}(\mathbf{A})$ DoF's. Since $\operatorname{rank}(\mathbf{A})=\operatorname{rank}(\mathbf{B})$ (See Appendix A), we conclude that the solutions to the original problem and those to the equivalent problem have the same DoF.

\section{APPENDIX C}

In this Appendix, we will demonstrate that the two cascaded relay channels cannot be estimated at the $\mathrm{DN}$ by setting $\left\{\mathbf{P}_{l}, 2 \leq l \leq L\right\}$ as the permutations of $\mathbf{P}_{1}$.

Without loss of generality, we let $\mathbf{P}_{1}=\mathbf{I}_{N_{r}}$ and thus $\left\{\mathbf{P}_{l}, 1 \leq l \leq L\right\}$ will be a set of permutation matrices. Denote $\mathbf{E}$ to be an all-one $N_{r} \times N_{r}$ square matrix, and then obviously

$$
\mathbf{P}_{l} \mathbf{E}=\mathbf{E P}_{l}, \quad 1 \leq l \leq L .
$$


Define $\mathbf{T}=a \mathbf{E}+b \mathbf{I}_{N_{r}}$, where $b \neq 0$ and $-N_{r} a$. Since $|\mathbf{T}|=$ $b^{N_{r}-1}\left(b+N_{r} a\right) \neq 0, \mathbf{T}$ is invertible. According to (A-6),

$$
\mathbf{P}_{l} \mathbf{T}=\mathbf{T P}_{l}, \quad 1 \leq l \leq L .
$$

which can be rewritten as

$$
\mathbf{P}_{l}=\mathbf{T P}_{l} \mathbf{T}^{-1}, \quad 1 \leq l \leq L .
$$

Equation (A-8) indicates that, for permutation matrices $\left\{\mathbf{P}_{l}, 1 \leq l \leq L\right\}, \mathbf{T}_{2}=\mathbf{T}$ and $\mathbf{T}_{1}=\mathbf{T}^{-1}$ are solutions to the equivalent problem in (13). Since $\mathbf{T}$ has two DoF's embodied in the unknown $a$ and $b$, respectively, relay channel estimation is infeasible according to Proposition 3. Intuitively, suppose that $\hat{\mathbf{H}}_{1}$ and $\hat{\mathbf{H}}_{2}$ are solutions to

$$
\mathbf{H}_{o, l}=\mathbf{H}_{2} \mathbf{P}_{l} \mathbf{H}_{1}, \quad 1 \leq l \leq L,
$$

and then, according to (A-8), $\mathbf{T}^{-1} \hat{\mathbf{H}}_{1}$ and $\hat{\mathbf{H}}_{2} \mathbf{T}$ are also solutions to (A-9). Since $\mathbf{T}$ has two DoF's, relay channel estimation is infeasible.

\section{APPENDIX D}

Suppose $K$ is the number of eigenvector clusters of $\left\{\mathbf{P}_{l}, 2 \leq\right.$ $l \leq L\}$. We will first show that $K=1$ is a necessary condition for feasible relay channel estimation by constructing a $\mathbf{T}_{2}$ with $K$ DoF's that satisfies (15) in the equivalent problem.

Construct

$$
\mathbf{U}=\left[\mathbf{e}_{11}, \mathbf{e}_{12}, \cdots, \mathbf{e}_{1 n_{1}} ; \cdots ; \mathbf{e}_{K 1}, \mathbf{e}_{K 2}, \cdots, \mathbf{e}_{K n_{K}}\right],
$$

as an eigenvector matrix of $\mathbf{T}_{2}$, where $\mathbf{e}_{k n}$ $\left(1 \leq k \leq K, 1 \leq n \leq n_{k}\right)$ denotes the $n$th base of the $n_{k}$-dimensional space, $S_{k}$, which is spanned by the $k$ th eigenvector cluster of each pilot matrix. Further let $S_{k}$ be the $k$ th eigenspace of $\mathbf{T}_{2}$ with the associated eigenvalue $\lambda_{k}$, i.e., $\forall \mathbf{v} \in S_{k}, \mathbf{T}_{2} \mathbf{v}=\lambda_{k} \mathbf{v}$. Define $\boldsymbol{\Lambda}_{k}=\lambda_{k} \mathbf{I}_{n_{k}}$, then,

$$
\mathbf{T}_{2}=\mathbf{U} \boldsymbol{\Sigma} \mathbf{U}^{-\mathbf{1}},
$$

where $\boldsymbol{\Sigma}=\operatorname{diag}\left\{\boldsymbol{\Lambda}_{1}, \boldsymbol{\Lambda}_{2}, \cdots, \boldsymbol{\Lambda}_{K}\right\}$ denotes the $N_{r} \times N_{r}$ diagonal eigenvalue matrix. According to the eigenvector grouping rule, for any $2 \leq l \leq L$, invertible transform $\mathbf{P}_{l}$ maps space $S_{k}$ to itself, i.e., $\forall \mathbf{v} \in S_{k}, \mathbf{P}_{l} \mathbf{v} \in S_{k}$. As a result, $\mathbf{P}_{l} \mathbf{U}$ is still an eigenvector matrix of $\mathbf{T}_{2}$ for any $2 \leq l \leq L$, which indicates that $\mathbf{T}_{2}$ in (A-11), which has $K$ DoF's embodied in $K$ independent eigenvalues, satisfies (15) and therefore is a solution to the equivalent problem. According to Proposition 3 , relay channel estimation is feasible if and only if the solutions to the equivalent problem have only one DoF. Thus we conclude that $K=1$ is a necessary condition for feasible relay channel estimation.

To show that $K=1$ is also a sufficient condition for feasible relay channel estimation, we suppose that when $K=1$, there exists an invertible $\mathbf{T}_{2} \neq \alpha \mathbf{I}_{2}$, where $\alpha$ denotes an arbitrary complex scalar, that satisfies (15). Since $\mathbf{T}_{2} \neq \alpha \mathbf{I}_{2}$, it has $J(J \geq 2)$ different eigenvalues corresponding to $J$ different eigenspaces, $S_{j}, 1 \leq j \leq J$. According to (15), if $\mathbf{U}$ is an eigenvector matrix of $\mathbf{T}_{2}$, so is $\mathbf{P}_{l} \mathbf{U}, 2 \leq l \leq L$. In other words, for any $2 \leq l \leq L$, transform $\mathbf{P}_{l}$ maps $S_{j}$ to itself, which means $S_{j}$ for any $j$ is a space spanned by eigenvectors of any pilot matrix, $\mathbf{P}_{l}$. According to the eigenvector grouping rule, the number of eigenvector clusters of $\mathbf{P}_{l}$ 's is greater than or equal to $J$, which is contradictory to the supposition that $K=1$. Therefore, $\mathbf{T}_{2}=\alpha \mathbf{I}_{2}$, where $\alpha$ denotes an arbitrary scalar, is the sole solution to (15) when $K=1$. According to Proposition 3, we conclude $K=1$ is also a sufficient condition for feasible relay channel estimation.

\section{APPENDIX E}

To prove that the design rules given in Section IV ensure feasible relay channel estimation, we will first show the following fact.

Fact 1. Any eigenvector of $\boldsymbol{P}_{3}$ does not have zero elements if $\boldsymbol{P}_{3}$ is an indivisible permutation of $\boldsymbol{P}_{2}$.

Proof: According to the design rules given in Section IV,

$$
\mathbf{P}_{2}=\left[\begin{array}{c}
\mathbf{r}_{1} \\
\mathbf{r}_{2} \\
\vdots \\
\mathbf{r}_{N_{r}}
\end{array}\right]=\left[\begin{array}{cccc}
\gamma_{1} & 0 & \cdots & 0 \\
0 & \gamma_{2} & \cdots & 0 \\
\vdots & \vdots & \ddots & \vdots \\
0 & 0 & \cdots & \gamma_{N_{r}}
\end{array}\right],
$$

where $\mathbf{r}_{i}$ denotes the $i$ th row vector of $\mathbf{P}_{2}$. Sine $\mathbf{P}_{3}$ is a permutation of $\mathbf{P}_{2}$, we have

$$
\mathbf{P}_{3}=\left[\mathbf{r}_{f^{-1}(1)}^{T}, \mathbf{r}_{f^{-1}(2)}^{T}, \cdots, \mathbf{r}_{f^{-1}\left(N_{r}\right)}^{T}\right]^{T},
$$

where $f^{-1}(i)=j$ if $i$ is directly reachable from $j$.

Suppose $\mathbf{v}=\left[v_{1}, v_{2}, \cdots, v_{N_{r}}\right]^{T}$ is a non-zero eigenvector of $\mathbf{P}_{3}$ with the associated eigenvalue, $\lambda$, and then $\mathbf{P}_{3} \mathbf{v}=\lambda \mathbf{v}$, i.e.,

$$
\mathbf{r}_{f^{-1}(i)} \cdot \mathbf{v}=\gamma_{f^{-1}(i)} v_{f^{-1}(i)}=\lambda v_{i}, \quad 1 \leq i \leq N_{r} .
$$

Suppose $v_{i}=0$ for an $i$. Since both $\mathbf{P}_{2}$ and $\mathbf{P}_{3}$ are unitary, $\gamma_{f^{-1}(i)} \neq 0$ and $\lambda \neq 0$. As a result, $v_{f^{-1}(i)}$ must be zero to satisfy (A-14). For the same reason, it can be shown that $v_{j}=0$ for any $j$ that reaches $i$. Since $\mathbf{P}_{3}$ is an indivisible permutation of $\mathbf{P}_{3}$, any $j \in\left\{1,2, \cdots, N_{r}\right\}$ reaches $i$. As a result, $\mathbf{v}$ must be a zero vector, which is contradictory to the above supposition. Thus we conclude that any eigenvector of $\mathbf{P}_{3}$ does not have zero elements.

Since $\mathbf{P}_{2}$ is a diagonal matrix with $N_{r}$ different non-zero elements, the standard bases of an $N_{r}$-dimensional space, $\mathbf{e}_{1}=$ $[1,0, \cdots, 0]^{T}, \mathbf{e}_{2}=[0,1, \cdots, 0]^{T}, \cdots, \mathbf{e}_{N_{r}}=[0,0, \cdots, 1]^{T}$, are the $N_{r}$ eigenvectors of $\mathbf{P}_{2}$. According to Fact 1, any eigenvector of $\mathbf{P}_{3}$ does not have zero elements and hence does not fall in any subspace spanned by a subset of the standard bases, $\left\{\mathbf{e}_{1}, \mathbf{e}_{2}, \cdots, \mathbf{e}_{N_{r}}\right\}$, which, according to the eigenvector grouping rule, means that $\mathbf{P}_{2}$ and $\mathbf{P}_{3}$ have only one eigenvector cluster. According to Proposition 4, we conclude that the design rules given in Section IV ensure feasible relay channel estimation.

\section{ACKNOWLEDGMENT}

The authors would like to thank Dr. Yibo Zhao for many valuable discussions on this paper. 


\section{REFERENCES}

[1] D. Soldani and S. Dixit, "Wireless relays for broadband access," IEEE Commun. Mag., vol. 46, pp. 58-66, Mar. 2008.

[2] S. W. Peters and R. W. Heath, "The future of WiMAX: multihop relaying with IEEE 802.16j," IEEE Commun. Mag., vol. 47, pp. 104-111, Jan. 2009.

[3] A. H. Ali and J. G. Gardiner, "The performance of repeaters in UMTS networks," in Proc. IEEE Mediterranean Electrotechnical Conf., vol. 2.

[4] J. Laneman, D. N. C. Tse, and G. W. Wornell, "Cooperative diversity in wireless networks: Efficient protocols and outage behavior," IEEE Trans. Inf. Theory, vol. 50, no. 12, pp. 3062-3080, Dec. 2004.

[5] A. Sendonaris, E. Erkip, and B. Aazhang, "User cooperation diversitypart I: System description," IEEE Trans. Commun., vol. 51, no. 11, pp. 1927-1938, Nov. 2003.

[6] A. Sendonaris, E. Erkip, and B. Aazhang, "User cooperation diversitypart II: Implementation aspects and performance analysis," IEEE Trans. Commun., vol. 51, no. 11, pp. 1939-1948, Nov. 2003.

[7] J. Laneman and G. W. Wornell, "Distributed space-time-coded protocols for exploiting cooperative diversity in wireless networks," IEEE Trans. Inf. Theory, vol. 49, no. 10, pp. 2415-2425, Oct. 2003.

[8] A. Nosratinia, T. E. Hunter, and A. Hedayat, "Cooperative communication in wireless networks," IEEE Commun. Mag., vol. 42, pp. 74-80, Oct. 2004.

[9] S. M. Kay, Fundamentals of Statistical Signal Processing: Estimation Theory. Upper Saddle River, NJ: Prentice-Hall PTR, 1998.

[10] A. J. Paulraj and C. B. Papadias, "Space-time processing for wireless communications," IEEE Signal Processing Mag., vol. 14, no. 6, pp. 49-83, Nov. 1997.

[11] J. H. Kotecha and A. M. Sayeed, "Transmit signal design for optimal estimation of correlated MIMO channels," IEEE Trans. Signal Processing, vol. 52, no. 2, pp. 546-557, Feb. 2004.

[12] G. Y. Li, N. Seshadri, and S. Ariyavisitakul, "Channel estimation for OFDM systems with transmitter diversity in mobile wireless channels," IEEE J. Select. Areas Commun., vol. 17, no. 3, pp. 461-471, Mar. 1999.

[13] C. S. Patel and G. L. Stuber, "Channel estimation for amplify and forward relay based cooperation diversity systems," IEEE Trans. Wireless Commun., vol. 6, no. 6, pp. 2348-2356, Jun. 2007.

[14] F. Gao, T. Cui, and A. Nallanathan, "On channel estimation and optimal training design for amplify and forward relay networks," IEEE Trans. Wireless Commun., vol. 7, no. 5, pp. 1907-1916, May. 2008.

[15] J. Ma, P. Orlik, J. Zhang, and G. Y. Li, "Pilot matrix design for interim channel estimation in two-hop MIMO AF relay systems," in Proc. IEEE Int. Conf. on Commun., June 2009.

[16] G. J. Foschini, "Layered space-time architechture for wireless communication in a fading environment when using multi-element antennas," Bell Labs Tech. J., vol. 1, no. 2, pp. 41-59, 1996.

[17] C. R. Rao and S. K. Mitra, Generalized Inverse of Matrices. New York: John Wiley and Sons, 1971.

[18] J. Brewer, "Kronecker products and matrix calculus in system theory," IEEE Trans. Circuits and Systems, vol. 25, no. 9, pp. 772-781, Sep. 1978.

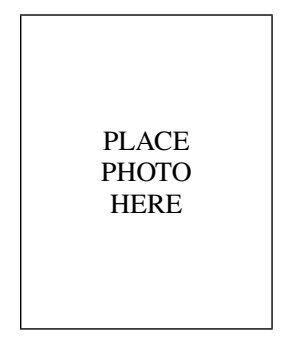

Jun Ma received his B.S. and M.S. degrees in 2003 and 2006, respectively, both from the Department of Electronic Engineering and Information Science, University of Science and Technology of China, Hefei, China, and his Ph.D. degree in 2010 from the Department of Electrical and Computer Engineering, Georgia Institute of Technology, Atlanta, GA.

From 2006 to 2010, he was a Research Assistant with the School of Electrical and Computer Engineering, Georgia Institute of Technology, under the supervision of Dr. Geoffrey Ye Li. He has held research intern positions with the Mitsubishi Electric Research Laboratory (MERL), Cambridge, MA, in 2007 and 2008, and the Wireless Systems Research Lab (WSRL) at Hitachi America Ltd., R\&D., Santa Clara, CA, in 2010, respectively. He is currently with Marvell Semiconductors Inc., Santa Clara, CA. His research interests include cognitive radio, cooperative communications, and high-mobility OFDM.

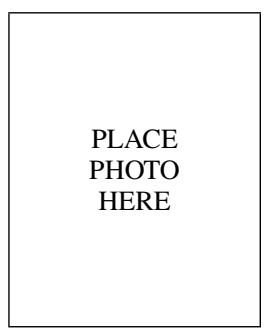

Philip V. Orlik (S'97, M'99) was born in New York, NY in 1972. He received the B.E. degree in 1994 and the M.S. degree in 1997 both from the State University of New York at Stony Brook. In 1999 he earned his $\mathrm{Ph}$. D. in electrical engineering also from SUNY Stony Brook.

$\mathrm{He}$ is currently the Team Leader of the Mobile Systems Group at Mitsubishi Electric Research Laboratories Inc. located in Cambridge, MA. His primary research focus is on advanced wireless and mobile cellular communications, sensor networks, ad hoc networking and UWB. Other research interests include vehicular/car-tocar communications, mobility modeling, performance analysis, and queuing theory.

\begin{tabular}{|l|}
\hline \\
\\
PLACE \\
PHOTO \\
HERE \\
\end{tabular}

Jinyun Zhang received her Ph.D. degree in electrical engineering from University of Ottawa, Canada in 1991. Dr. Zhang then joined Nortel Networks, where she held various management positions and engineering positions of increasing responsibility in the areas of digital signal processing, wireless communication and optical networks. Since 2001, Dr. Zhang has been the Manager of the Digital Communications \& Networking Group at Mitsubishi Electric Research Laboratories (MERL), Cambridge, MA, USA. She is a MERL Fellow and leading various new research projects on broadband wireless communications, optical communications, advanced signal processing, smart grid technologies and standardization activities.

Dr. Zhang has authored and co-authored more than 170 publications, invented and co-invented more than 140 patents and patent applications, and made numerous contributions to international wireless communications standards. Dr. Zhang is a Fellow of the IEEE and a member of the IEEE BT, COMM, IT, ITS, SP, and VT Socieites. She is an Associate Editor of IEEE Transactions on Broadcasting, and has served as a Technical Program Committee member for various IEEE international conferences.

\begin{tabular}{|c|}
\hline \\
PLACE \\
PHOTO \\
HERE \\
\end{tabular}

Geoffrey Ye Li received his B.S.E. and M.S.E. degrees in 1983 and 1986, respectively, from the Department of Wireless Engineering, Nanjing Institute of Technology, Nanjing, China, and his Ph.D. degree in 1994 from the Department of Electrical Engineering, Auburn University, Alabama.

He was a Teaching Assistant and then a Lecturer with Southeast University, Nanjing, China, from 1986 to 1991, a Research and Teaching Assistant with Auburn University, Alabama, from 1991 to 1994, and a Post-Doctoral Research Associate with the University of Maryland at College Park, Maryland, from 1994 to $1996 . \mathrm{He}$ was with AT\&T Labs - Research at Red Bank, New Jersey, as a Senior and then a Principal Technical Staff Member from 1996 to 2000. Since 2000, he has been with the School of Electrical and Computer Engineering at Georgia Institute of Technology as an Associate and then a Full Professor. He is also holding the Cheung Kong Scholar title at the University of Electronic Science and Technology of China since March 2006.

His general research interests include statistical signal processing and telecommunications, with emphasis on OFDM and MIMO techniques, crosslayer optimization, and signal processing issues in cognitive radios. In these areas, he has published over 200 papers in refereed journals or conferences and two books, 20 of which are with over 100 Google citations. He has over 20 patents granted or filed. He once served or is currently serving as an editor, a member of editorial board, and a guest editor for over 10 technical journals. $\mathrm{He}$ organized and chaired many international conferences, including technical program vice-chair of IEEE ICC'03 and co-chair of IEEE SPARC'11. He has been awarded an IEEE Fellow for his contributions to signal processing for wireless communications since 2006, selected as a Distinguished Lecturer for $2009-2010$ by IEEE Communications Society, and won 2010 IEEE Communications Society Stephen O. Rice Prize Paper Award in the field of communications theory. 